\title{
Avaliação de propriedades termoquímicas e físico-mecânicas de três espécies florestais de rápido crescimento
}

\author{
Evaluation of thermochemical and physico- \\ mechanical properties of three fast growing forest \\ species
}

Henrique Römer Schulz ${ }^{1}$, Andrey Pereira Acosta ${ }^{1}$, Ezequiel Gallio ${ }^{1}$, Kelvin Techera Barbosa ${ }^{2}$, Darci Alberto Gatto ${ }^{2}$

\begin{abstract}
${ }^{1}$ UFPel - Universidade Federal de Pelotas, Centro de Desenvolvimento Tecnológico (CDTec), Rua Gomes Carneiro, 1, CEP: 96010-610, Campus Anglo, Pelotas, Rio Grande do Sul, Brasil.

${ }^{2}$ UFPel - Universidade Federal de Pelotas, Centro de Engenharias (CEng), Rua Benjamin Constant, CEP: 96010-020, Campus Cotada, Pelotas, Rio Grande do Sul, Brasil.

e-mail: henriqueschulz09@hotmail.com, egeng.florestal@gmail.com, andreysvp@gmail.com,darcigatto@yahoo.com
\end{abstract}

\section{RESUMO}

As madeiras apresentam propriedades tecnológicas distintas, por ser material orgânico e heterogêneo, sendo necessário uma avaliação para o uso adequado. Sendo assim, o objetivo deste trabalho foi a avaliação de propriedades tecnológicas, sendo elas: massa especifica básica, massa especifica aparente a 12\%, teor de umidade de equilíbrio, flexão estática (MOE e MOR), compressão paralela as fibras (Fc e Ec), análise colorimétrica, termogravimetria (TGA e DTG) e fourier transform infrared spectroscopy (FT-IR), das espécies de Corymbia maculata, Eucalyptus grandis, Pinus elliottii. Determinaram-se as propriedades tecnológicas conforme descrito nas normas ASTM D 2395-17 (2017) e ASTM D 143-94 (2014). Em relação as propriedades físico-mecânicas, as species apresentaram diferenças significativas baseadas em análises estatisticas, possuindo destaque a espécie de Corymbia maculata. Em relação a análise colorimétrica, foi verificado que a espécie Corymbia maculata possui uma coloração marrom-avermelhado, a espécie de Pinus elliottii amarelo com tonalidade clara, enquanto o Eucalyptus grandis avermelhada com tonalidade clara. Para o FT-IR foi constatado picos semelhantes entre as espécies, porém com intensidades distintas, sendo estes referentes as vibrações moleculares características dos componentes orgânicos da madeira. Por meio da análise termogravimétrica percebe-se que as espécies de Pinus elliottii e Corymbia maculata, apresentaram maior estabilidade térmica, quando comparadas ao Eucalyptus grandis, possuindo um menor percentual de perda de massa e um retardo de temperatura em relação ao pico máximo de degradação (DTG), próximo aos $400^{\circ} \mathrm{C}$, sendo essas propriedades analisadas com testes ANOVA com significância de $5 \%$. Sendo assim destaca-se uma heterogeneidade entre as espécies avaliadas nesse estudo, tanto do ponto de vista físico, mecânico, térmico e químico, fazendo-se necessário a caracterização de tais propriedades para o emprego correto destes materiais.

Palavras-chave: Degradação térmica, FT-IR, Eucalyptus, Pinus, Dureza.

\begin{abstract}
The woods have distinct technological properties, as they are organic and heterogeneous material, requiring an assessment for proper use. Therefore, the objective of this work was the evaluation of technological properties, which are: basic density, apparent density at $12 \%$, equilibrium moisture content, static flexion (MOE and MOR), parallel compression of the fibers (Fc and Ec), colorimetric analysis, thermogravimetry (TGA and DTG) and Fourier transform infrared spectroscopy (FT-IR), of the species of Corymbia maculata, Eucalyptus grandis, Pinus elliottii. The technological properties were determined as described in the ASTM D 2395-17 (2017) and ASTM D 143-94 (2014) standards. Regarding the physical-mechanical properties, the species showed significant differences based on statistical analysis, highlighting the species of Corymbia maculata. Regarding the colorimetric analysis, it was found that the species Corymbia maculata has a reddish-brown color, the Pinus elliottii species loves it with a light shade, while the Eucalyptus grandis reddish with a
\end{abstract}


light shade. For FT-IR, similar peaks were found between species, but with different intensities, these being related to the molecular vibrations characteristic of the organic components of wood. Through thermogravimetric analysis, it can be seen that the species of Pinus elliottii and Corymbia maculata, presented greater thermal stability when compared to Eucalyptus grandis, having a lower percentage of mass loss and a delay in temperature in relation to the maximum peak of degradation ( DTG), close to $400^{\circ} \mathrm{C}$, and these properties were analyzed with ANOVA tests with $5 \%$ significance. Thus, there is a heterogeneity among the species evaluated in this study, both from a physical, mechanical, thermal and chemical point of view, making it necessary to characterize these properties for the correct use of these materials.

Keywords: Thermal degradation, FT-IR, Eucalyptus, Pinus, Hardness.

\section{INTRODUÇÃO}

O desenvolvimento do Brasil no cultivo de madeira reflorestada especialmente os gêneros Eucalyptus e Pinus tem sido amplamente estudado e utilizado pelo setor industrial madeireiro. Neiva [1] e Ballesteros [2], constatam que as utilizações da madeira podem ser, para uso estrutural, móveis, madeira serrada, dormentes, uso energético, celulose e papel.

A madeira por ser um material heterogêneo, necessita da avaliação de suas propriedades tecnológicas, sendo estas, propriedades físicas, mecânicas, químicas, térmicas. Muller [3] e Lahr [4], constatam que a caracterização das propriedades físicos-mecânicas é de grande importância para o uso estrutural. As propriedades da madeira que determinam a sua qualidade (por exemplo, propriedades fícias e químicas) são o resultado do crescimento e formação da árvore [5]. Conforme estas variações citadas ocorrem há alterações em determinadas propriedades na madeira, podendo ser térmicas, colorimétricas e mecânicas. Mori [6], cita que a cor está ligada a qualidade do material e questões estéticas ao qual vai ser empregado, sendo que há variação entre espécies. Para Carneiro [7], a análise térmica se faz necessário visto que a madeira é empregada no uso para geração de energia, sendo que a madeira é um material de origem orgânica e um material sólido inflamável.

Uma técnica que facilita a caracterização da madeira é o FT-IR (espectroscopia no infravermelho com transformada de Fourier), conforme Gallio [8], relata que a espectroscopia FT-IR é uma importante ferramenta para avaliação de componentes orgânicos na madeira, visto que, cada molécula vibra em um número de onda específico. Ainda Ojeda \& Dittrich [9], destaca que cada material apresenta diferentes intensidades de ligações ou estrutura, portanto, exibirá um espectro diferente na espectroscopia FTIR.

Devido a necessidade de avaliação das propriedades tecnológicas de madeiras de reflorestamento com potencial, o presente trabalho teve como objetivo analisar as propriedades, massa especifica básica, massa especifica aparente a 12\%, teor de umidade de equilíbrio, flexão estática (Mor e Moe), compressão paralela ás fibras $\left(\mathrm{F}_{\mathrm{c}}\right.$ e $\left.\mathrm{E}_{\mathrm{c}}\right)$, análise colorimétrica, análise termogravimétrica (TGA e DTG) e espectroscopia no infravermelho com transformada de Fourier (FT-IR), para as espécies Corymbia maculata, Eucalyptus grandis e Pinus ellliottii, que são madeiras de reflorestamento com grande potencial no setor madeireiro.

\section{MATERIAIS E MÉTODOS}

\subsection{Seleção e Obtenção do Material}

As espécies utilizadas foram, Corymbia maculata, Eucalyptus grandis, Pinus elliottii, sendo as espécies comidade de 22 anos aproximadamente. Posteriormente, das tábuas provenientes das espécies selecionadas, foram confeccionados os corpos de prova $(\mathrm{CP})$, de acordo com a tabela 1:

Tabela 1: Dimensões dos corpos de prova (CP) para as análises tecnológicas.

\begin{tabular}{c|c|c|c|c}
\hline \multirow{2}{*}{ ANÁLISES } & \multicolumn{2}{|c|}{ PLANO ANATÔMICO - DIMENSÕES (mm) } & NÚMERO DE AMOSTRAS \\
\cline { 2 - 4 } & TANGENCIAL & RADIAL & LONGITUDINAL & POR ESPÉCIE \\
\hline Flexão Estática & 20 & 20 & 330 & 6 \\
\hline Compressão Paralela & 20 & 20 & 80 & 6 \\
\hline Dureza Janka & 20 & 20 & 80 & 6 \\
\hline Massa específica básica & 20 & 20 & 100 & 6 \\
\hline Massa específica aparente a 12\% & 20 & 20 & 100 & Unitário \\
\hline FT-IR & \multicolumn{3}{|c|}{ Amostras moídas } & Unitário \\
\hline TGA & \multicolumn{3}{c}{ Amostras moídas } \\
\hline
\end{tabular}


Depois de dimensionados, os corpos de prova foram acondicionados em câmara climatizada (temperatura de $20^{\circ} \mathrm{C}$ e $65 \%$ de umidade relativa) até massa constante e um teor de umidade de $12 \%$.

\subsection{Amostras na condição seca}

Para certas análises as amostras tinham que estar nas condições de seca, ou seja, teor de umidade próximo de $0 \%$, para essa condição ser atendida as amostras foram colocadas na estufa de escala laboratorial (capacidade de 100 litros e temperatura máxima de $\left.300^{\circ} \mathrm{C}\right)$, primeiramente a $50^{\circ} \mathrm{C}\left( \pm 3^{\circ} \mathrm{C}\right)$ e alcançando a temperatura de $100^{\circ} \mathrm{C}\left( \pm 3^{\circ} \mathrm{C}\right)$ por um período de 48 horas.

\subsection{Caracterização das Propriedades físicas}

\subsubsection{Massa especifica básica e massa especifica aparente a $12 \%$}

A massa específica básica $\left(\rho_{\text {Bás }}\right)$ e aparente a $12 \%\left(\rho_{12 \%}\right)$ foi determinada por meio da adaptação da normatização da American Society for Testing and Materials D 2395 [10]. Utilizaram-se 10 corpos de prova os quais foram mensurados com auxílio de um paquímetro digital (com resolução de $0,01 \mathrm{~mm}$ ) e balança analítica (com resolução de $0,001 \mathrm{~g}$ ), foram medidas as dimensões e massa dos corpos de prova e posteriormente por meio das equações (1 e 2), caracterizou-se as mesmas.

$$
\begin{aligned}
& \rho_{B a ́ s}=\left(\frac{\mathrm{M}_{0}}{\mathrm{v}_{\text {Saturado }}}\right) \\
& \rho_{12 \%}=\left(\frac{\mathrm{M}_{12 \%}}{\mathrm{v}_{12 \%}}\right)
\end{aligned}
$$

Em que: $\rho_{\text {Bás }}=$ massa especifica básica $\left(\mathrm{g} / \mathrm{cm}^{3}\right) ; \mathrm{M}_{0}=$ massa seca em estufa a $100^{\circ} \mathrm{C}\left( \pm 3^{\circ} \mathrm{C}\right)(\mathrm{g}) ; \mathrm{V}_{\text {Saturado }}=$ volume com a amostra saturada $\left(\mathrm{cm}^{3}\right) ; \rho_{12 \%}=$ massa especifica aparente a $12 \%$ de teor de umidade $\left(\mathrm{g} / \mathrm{cm}^{3}\right) ; \mathrm{M}_{12 \%}=$ massa a $12 \%$ (g); $\mathrm{V}_{12 \%}=$ volume a $12 \%\left(\mathrm{~cm}^{3}\right)$.

\subsubsection{Teor de Umidade de Equilíbrio}

O teor de umidade de equilíbrio foi mensurado, com auxílio de uma balança analítica (com resolução de 0,001g), utilizou-se a adaptação da normatização ASTM D 2395 [10]. Utilizaram-se 10 corpos de prova, os quais foram medidos as massas dos corpos de prova com teor de umidade de aproximadamente $12 \%$ e nas condições de amostras secas, por meio da equação (3), caracterizou se a mesma.

$$
\mathrm{TU}_{\text {Equilibrio }}=\left(\frac{\mathrm{M}_{12 \%}-\mathrm{M}_{0}}{\mathrm{M}_{0}}\right) * 100
$$

Em que: $\mathrm{M}_{12 \%}=$ massa na condição de $12 \%$ de Teor de Umidade $(\mathrm{g}) ; \mathrm{M}_{0}=$ massa seca em estufa a $100^{\circ} \mathrm{C}\left( \pm 3^{\circ} \mathrm{C}\right)$ $(\mathrm{g}) ; \mathrm{TU}_{\text {Equilibrio }}=$ Teor de umidade de equilíbrio (\%).

\subsection{Caracterizações das Propriedades mecânicas}

Para as propriedades mecânicas foram utilizados 10 corpos de prova por caracteriazção em uma máquina universal de ensaios EMIC, com capacidade de carga de 300kN, sendo baseado em técnicas normativas ASTM D143-94 [11]. As leituras de carga e propriedades referentes a resistência para cada teste, foram obtidas através de um sistema informatizado de aquisição de dados e transferidas para uma planilha eletrônica (Software Tesc).

O teste de flexão estática da madeira foi realizado, através da aplicação de uma força no centro da amostra que estava bi apoiada, deste teste foram obtidos a módulo de ruptura e módulo de elasticidade. O ensaio foi conduzido com velocidade de $1,3 \mathrm{~mm} / \mathrm{min}$ e a célula de carga que possui capacidade para 3 toneladas, sendo o vão 14 vezes o eixo anatômico radial.

O teste de Dureza Janka da madeira foi realizado com a verificação do esforç, necessário para introduzir uma semiesfera de aço com $1,13 \mathrm{~cm}^{2}$ de diâmetro, sendo feito uma leitura em cada face, sendo elas, tangencial, radial e topo.

O teste de compressão paralela às fibras da madeira foi realizado com a verificação do esforço, em Mpa, de um cabeçote plano no topo das amostras, obtendo os valores de módulo de ruptura $\left(\mathrm{F}_{\mathrm{c}}\right)$ e elasticidade $\left(\mathrm{E}_{\mathrm{c}}\right)$. 


\subsection{Análise colorimétrica}

Para a colorimetria, efetuaram-se seis leituras para cada corpo de prova (três na superfície radial e três na tangencial, utilizando-se 6 amostras para cada espécie analisada). Para a análise, fez-se uso de um colorímetro Konica Minolta, modelo CR-400, com fonte iluminante D65 e ângulo de observação de $10^{\circ}$.

O aparelho forneceu os seguintes parâmetros colorimétricos: luminosidade $\left(\mathrm{L}^{*}\right)$, coordenada vermelho-verde ou matriz vermelho $\left(\mathrm{a}^{*}\right)$, coordenada azul-amarelo ou matriz amarelo $\left(\mathrm{b}^{*}\right)$, saturação da cor ( $\left.\mathrm{c}^{*}\right)$ e o ângulo de tinta (h*), baseados no sistema de cor CIELa*b* 1976. Para determinar as alterações de cor, foi utilizada a Equação (4).

$$
\Delta \mathrm{E}=\sqrt{\Delta \mathrm{L}^{2}+\Delta \mathrm{a}^{2}+\Delta \mathrm{b}^{2}}(4)
$$

Em que: $\Delta \mathrm{E}$ - variação total da cor das madeiras; $\Delta \mathrm{L}$ - variação do gradiente de claridade; $\Delta \mathrm{a}$ - variação do gradiente da coordenada vermelho-verde; $\Delta \mathrm{b}$ - variação do gradiente da coordenada azul-amarelo.

\subsection{Espectroscopia no infravermelho com transformada de Fourier(FT-IR)}

A análise de FT-IR, via ATR, foi feita através do equipamento modelo FT/IR-4100, com 32 varreduras por espécie analisada, com um número de onda de 2000 a $800 \mathrm{~cm}^{-1}$, resolução de $4 \mathrm{~cm}^{-1}$, a velocidade do scanner de $2 \mathrm{~mm} / \mathrm{sec}$, filtro de $30000 \mathrm{~Hz}$.

\subsection{Análise termogravimétrica (TGA) e (DTG)}

Foi efetuada a análise termogravimétrica no equipamento NAVAS TGA 1000, com uma análise para cada uma das três espécies estudadas, com uma vazão constante de $2 \mathrm{~L} \cdot \mathrm{min}^{-1}$. As curvas termogravimétricas foram obtidas a partir de $30^{\circ} \mathrm{C}$ até a temperatura máxima de $600{ }^{\circ} \mathrm{C}$, com taxa de aquecimento de $10^{\circ} \mathrm{C} \cdot \mathrm{min}^{-1}$. Para tanto foi avaliada a curva termogravimétrica (TGA) perda de massa em função da temperatura e posteriormente foi utilizada a primeira derivada da curva (DTG).

\subsection{Análise estatística dos resultados das propriedades}

Primeiramente foram coletados os dados obtidos através dos testes físicos normatizados e posteriormente foram feitas análises descritivas, sendo estas: médias, desvio padrão, coeficiente de variação, sendo as médias comparadas, posteriormente, por meio do teste LSD de Fisher, com 5\% de probabilidade de erro.

\section{RESULTADOS E DISCUSSÃO}

A tabela 2 traz parâmetros de massa específica aparente a 12\%, massa específica básica e teor de umidade das três espécies estudadas. Nota-se que para ambas massa especifica, a espécie de C. maculata obteve valores superiores, sendo esses significativos. Tal espécie apresentou uma superioridade de 93,47\% quando comparado ao E. grandis e de 71,15\% quando comparado ao $P$. elliottii para a $\rho_{\text {Bás }}$, sendo semelhante para a $\rho_{12 \%}$.

Tabela 2: Estatística descritiva para massa especifica aparente a 12\%, massa especifica básica e Teor de umidade de equilíbrio.

\begin{tabular}{|c|c|c|c|c|c|c|}
\hline \multirow[t]{2}{*}{ AMOSTRAS } & \multicolumn{2}{|c|}{$\begin{array}{c}\text { MASSA ESPECIFICA } \\
\text { APARENTE A } 12 \%\left(\mathrm{~g} / \mathrm{cm}^{3}\right)\end{array}$} & \multicolumn{2}{|c|}{$\begin{array}{l}\text { MASSA ESPECIFICA } \\
\text { BÁSICA }\left(\mathbf{g} / \mathrm{cm}^{3}\right)\end{array}$} & \multicolumn{2}{|c|}{$\begin{array}{l}\text { TEOR DE UMIDADE DE } \\
\text { EQUILÍBRIO (\%) }\end{array}$} \\
\hline & MÉDIA & $\mathrm{CV}$ & MÉDIA & $\mathrm{CV}$ & MÉDIA & $\mathrm{CV}$ \\
\hline C. maculata & $\begin{array}{l}0,89 \mathrm{c} \\
(0,03)\end{array}$ & 3,76 & $\begin{array}{l}0,76 \mathrm{c} \\
(0,01)\end{array}$ & 1,63 & 10,65 a $(0,09)$ & 0,82 \\
\hline E. grandis & $\begin{array}{l}0,46 \mathrm{a} \\
(0,05)\end{array}$ & 10,15 & $\begin{array}{c}0,40 \mathrm{a} \\
0,04)\end{array}$ & 8,54 & $10,56 \mathrm{a}(0,11)$ & 1,02 \\
\hline P. elliotti & $\begin{array}{l}0,52 \mathrm{~b} \\
(0,04)\end{array}$ & 6,92 & $\begin{array}{l}0,45 \mathrm{~b} \\
(0,03)\end{array}$ & 6,18 & 11,39 a $(0,06)$ & 0,50 \\
\hline $\mathrm{F}$ & $207,09 *$ & - & $274,50 *$ & - & $137,45 *$ & - \\
\hline
\end{tabular}

Em que: Valores entre parênteses = Desvio Padrão na unidade da medida de cada propriedade; Letras diferentes, nas colunas, indicam diferenças significativas entre as espécies, conforme o teste LSD Fisher, em 95\% de confiança; CV = coeficiente de variação $(\%)$; * significativo em $5 \%$ de probabilidade de erro, pelo teste $\mathrm{F}(\mathrm{p}<0,05)$. 
Essa superiodade nos valores médio esta associado a espécie de C.maculata possuir maior quantidade de material lenhoso por unidade de volume, quando comparado as demais.Martins [12] ao analisar a madeira de C. maculata aos 21 anos de idade, obteve valores para densidade básica em torno de $0,805 \mathrm{~g} / \mathrm{cm}^{3}$, valor pouco superior ao encontrado no presente estudo, isto pode ser justificado pela posição em que se foi extraído os corpos de prova. A massa especifica básica média do Eucalyptus grandis foi de $0,40 \mathrm{~g} / \mathrm{cm}^{3}$, Gonçalez et al. [13]. Silva et al. [14] e Batista [15], ao analisar espécies do gênero Eucalyptus encontraram valores próximosde $0,45 \mathrm{~g} / \mathrm{cm}^{3}$ para a massa especifica.

Para a madeira de P.elliottii percebe-se que o valor encontrado no presente estudo $\left(0,45 \mathrm{~g} / \mathrm{cm}^{3}\right)$ vai de acordo com o encontrado por Vidaurre [16] ao analisar a madeira do gênero Pinus com 15 anos de idade obteve valores médios de 0,459 $\mathrm{g} / \mathrm{cm}^{3}$.

Os valores médios de dureza Janka para as espécies analisadas (tabela 3), apresentaram diferença significativa nas diferentes faces das amostras entre as espécies, possuindo a C.maculata o maior valor, sendo significativo, com cerca de $168 \%$ para a face tangencial, $148 \%$ aproximadamente para radial e $122 \%$ para transversal, quando comparado a amostra de P.elliiotii que obteve o menor valor médio.

Tabela 3: Estatística descritiva para dureza Janka nas diferentes faces das amostras.

\begin{tabular}{c|c|c|c|c|c|c}
\hline \multirow{3}{*}{ AMOSTRAS } & \multicolumn{2}{|c|}{$\begin{array}{c}\text { DUREZA JANKA } \\
\text { TANGENCIAL }\end{array}$} & \multicolumn{2}{c|}{$\begin{array}{c}\text { DUREZA JANKA RADIAL } \\
\text { TENSÃO MÁXIMA (MPa) }\end{array}$} & \multicolumn{2}{c}{$\begin{array}{c}\text { DUREZA JANKA } \\
\text { TRANSVERSAL } \\
\text { TENSÃO MÁXIMA (MPa) }\end{array}$} \\
\cline { 2 - 7 } C. maculata & MÉDIA & CV & MÉDIA & CV & MÉDIA & CV \\
\hline \multirow{2}{*}{ E. grandis } & $\begin{array}{c}57,08 \mathrm{~b} \\
(3,31)\end{array}$ & 5,80 & $\begin{array}{c}56,04 \mathrm{~b} \\
(3,31)\end{array}$ & 9,53 & $\begin{array}{c}55,52 \mathrm{c} \\
(2,96)\end{array}$ & 5,34 \\
\hline P. elliotti & $\begin{array}{c}21,10 \mathrm{a} \\
(5,14)\end{array}$ & 24,38 & $\begin{array}{c}22,56 \mathrm{a} \\
(5,14)\end{array}$ & 16,56 & $\begin{array}{c}36,00 \mathrm{~b} \\
(6,38)\end{array}$ & 17,72 \\
\hline F & $\begin{array}{c}21,29 \mathrm{a} \\
(2,27)\end{array}$ & 10,65 & $\begin{array}{c}18,29 \mathrm{a} \\
(2,27)\end{array}$ & 6,11 & $\begin{array}{c}24,93 \mathrm{a} \\
(3,76)\end{array}$ & 15,09 \\
\hline
\end{tabular}

Em que: Valores entre parênteses = Desvio Padrão na unidade da medida de cada propriedade; Letras diferentes, nas colunas, indicam diferenças significativas entre as espécies, conforme o teste LSD Fisher, em 95\% de confiança; CV = coeficiente de variação (\%); * - significativo em 5\% de probabilidade de erro, pelo teste $\mathrm{F}(\mathrm{p}<0,05)$.

Xavier [17], em seu estudo analisando a tensão máxima do teste de dureza Janka no plano tangencial, constatou valores de 25,32 MPa, e 47,40 MPa, para as espécies de Eucalyptus grandis e Corymbia citriodora respectivamente, o que sugere valores próximos ao encontrado. Modes [18], constatou que os valores médios de dureza Janka da face transversal são geralmente maiores que os da face tangencial e radial. Isso ocorre devido à disposição dos elementos anatômicos que constituem a madeira, sendo esse fator determinante para a madeira de C.maculata possuir maior valor quando comparada as demais.

A Tabela 4 apresenta os resultados médios dos ensaios de flexão estática e compressão paralela às fibras, para as três espécies estudadas. Tais valores apresentam diferença significativa estatisticamente, sendo a espécie de C.maculata os maiores valores médios, MOE (103\%), MOR (119\%), $\mathrm{E}_{\mathrm{c}}(84 \%)$ e $\mathrm{F}_{\mathrm{c}}(75 \%)$, quando comparado a espécie de E.grandis que apresentou os menores valores médios.

Tabela 4: Estatística descritiva para Flexão estática e compressão paralela às fibras.

\begin{tabular}{c|c|c|c|c|c|c|c|c}
\hline \multirow{2}{*}{ AMOSTRAS } & \multicolumn{4}{|c|}{ FLEXÃO ESTÁTICA } & \multicolumn{3}{c}{ COMPRESS̃̃O PARALELA ÀS FIBRAS } \\
\cline { 2 - 9 } & MOE (MPa) & $\mathbf{C V}(\%)$ & $\begin{array}{c}\text { MOR } \\
\mathbf{( M P a}\end{array}$ & $\mathbf{C V}(\%)$ & $\mathbf{E}_{\mathbf{c}} \mathbf{( M P a )}$ & $\mathbf{C V}(\%)$ & $\begin{array}{c}\mathbf{F}_{\mathbf{c}} \\
\mathbf{( M P a})\end{array}$ & $\mathbf{C V}(\%)$ \\
\hline C. maculata & $\begin{array}{c}18001,2 \mathrm{~b} \\
(1674,06)\end{array}$ & 9,30 & $\begin{array}{c}149,0 \mathrm{~b} \\
(8,78)\end{array}$ & 5,89 & $\begin{array}{c}25664,0 \mathrm{c} \\
(3298,55)\end{array}$ & 12,85 & $\begin{array}{c}67,27 \mathrm{~b} \\
(5,00)\end{array}$ & 7,43 \\
\hline E. grandis & $\begin{array}{c}8867,45 \mathrm{a} \\
(752,84)\end{array}$ & 8,49 & $\begin{array}{c}68,02 \mathrm{a} \\
(8,70)\end{array}$ & 12,79 & $\begin{array}{c}13161,6 \mathrm{a} \\
(1963,42)\end{array}$ & 14,92 & $\begin{array}{c}38,42 \mathrm{a} \\
(2,43)\end{array}$ & 6,32 \\
\hline
\end{tabular}




\begin{tabular}{c|c|c|c|c|c|c|c|c}
\hline P. elliotti & $\begin{array}{c}9811,69 \mathrm{a} \\
(1354,48)\end{array}$ & 13,80 & $\begin{array}{c}74,46 \mathrm{a} \\
(9,27)\end{array}$ & 9,27 & $\begin{array}{c}17542,3 \mathrm{~b} \\
(3786,49)\end{array}$ & 21,58 & $\begin{array}{c}39,35 \mathrm{a} \\
(3,94)\end{array}$ & 10,02 \\
\hline $\mathrm{F}$ & $87,27 *$ & - & $152,66 *$ & - & $20,10 *$ & - & $104,20 *$ & - \\
\hline
\end{tabular}

Em que: Valores entre parênteses = Desvio Padrão na unidade da medida de cada propriedade; Letras diferentes, nas colunas, indicam diferenças significativas entre as espécies, conforme o teste LSD Fisher, em 95\% de confiança; CV = coeficiente de variação; $\mathrm{MOE}=$ Módulo de elasticidade; $\mathrm{MOR}=$ Módulo de Ruptura; $\mathrm{E}_{\mathrm{c}}=$ Elasticidade a compressão; $\mathrm{F}_{\mathrm{c}}=$ Resistência a compressão; * - significativo em $5 \%$ de probabilidade de erro, pelo teste $\mathrm{F}(\mathrm{p}<0,05)$.

Verifica-se que os resultados obtidos para a espécie de E. grandis estão de acordo com Gonçalves et al. [19], ao analisarem a madeira de $E$. urophylla $\times$ E. grandis, com cerca de 6 anos de idade, observaram resistência à flexão estática variando de 82,95 a 83,21 MPa, e módulo de elasticidade entre 9652 e $9798 \mathrm{MPa}$, o que vai de acordo com o observado no presente estudo. Gonzales [20] ao analisar a madeira de Pinus caribaea var. hondurensis com cerca de 22 anos de idade observou para o ensaio de flexão estática 83,77 MPa (MOR) e 7526,67 MPa (MOE).

Veiga [21] notou ao analisar a madeira de Corymbia maculata com 7 anos de idade com uma densidade básica de $0,705 \mathrm{~g} / \mathrm{cm}^{3}$ um módulo de elasticidade de $9116 \mathrm{MPa}$, valor muito inferior ao encontrado no presente estudo, isso se justifica pela diferença de idade, sendo a espécie aqui analisada com 22 anos de idade.

Leite [22] analisando a madeira de Corymbia maculata com 24 anos de idade obteve valores de 127 Mpa até 129 MPa para o Módulo de ruptura e $22120 \mathrm{MPa}$ até 25614 Mpa de módulo de elasticidade, sendo estes valores mais aproximados ao presente estudos, ressaltando a influência da idade nas propriedades mecânicas da madeira.

Percebe-se que tanto para o $\mathrm{E}_{\mathrm{c}}$ quanto para $\mathrm{F}_{\mathrm{c}}$, quando analisado ao teste de compressão paralela às fibras, a madeira de C.maculata possui destaque quando comparado as demais espécies estudadas, isto está associado diretamente a densidade básica do material. Haselein et al. [23] e Lobão [24], constatam que a densidade tem influência direta com as propriedades mecânicas do material.

Lima \& Garcia [25] ao avaliar a madeira de E. grandis aos 21 anos de idade para o $\mathrm{F}_{\mathrm{c}}$, verificaram valores que variaram de acordo com a densidade e posição em que os corpos de provas foram extraídos da árvores entre 35,23 para menor densidade e 61,09 MPa para maior densidade, valores esses que vão de acordo com o observado no presente estudo.

Serpa [26] ao avaliar a madeira de P.elliottii em relação ao $\mathrm{F}_{\mathrm{c}}$ encontrou um valor médio de $50 \mathrm{Mpa}$. Leite [22], ao analisar a madeira de $C$ maculata com cerca de 24 anos de idade observou um $\mathrm{F}_{\mathrm{c}}$ de $59 \mathrm{Mpa}$.

A Tabela 5 apresenta os parâmetros estatísticos colorimétricos nos planos anatômicos tangencial e radial sucessivamente, nota-se uma variação entre as três espécies da coordenada L* (luminosidade), onde P. elliottii demostrou maior valor e C. maculata o menor valor. Para as demais variáveis colorimétricas analisadas ( $\mathrm{a}^{*}, \mathrm{~b}^{*}, \mathrm{C}^{*}, \mathrm{~h}$ e $\left.\Delta \mathrm{E}\right)$, também se constatou variações significativas, aonde a maior variação de $\operatorname{cor}(\Delta \mathrm{E})$ foi observada entre $P$. elliottii (considerada espécie controle) e C. maculata.

Tabela 5: Resumo estatístico e valores médios para os parâmetros relacionados a colorimetria no eixo tangencial e radial.

\begin{tabular}{|c|c|c|c|c|c|c|}
\hline \multicolumn{7}{|c|}{ FACE TANGENCIAL } \\
\hline AMOSTRAS & $L^{*}$ & $A^{*}$ & $\mathrm{~B}^{*}$ & $C^{*}$ & $\mathbf{H}^{\circ}$ & \\
\hline C. maculata & $\begin{array}{c}64,48 \mathrm{a} \\
(1,44)\end{array}$ & $\begin{array}{l}5,71 \mathrm{~b} \\
(0,22)\end{array}$ & $\begin{array}{c}19,17 \text { a } \\
(0,91)\end{array}$ & $\begin{array}{c}20,00 \mathrm{a} \\
(0,92)\end{array}$ & $\begin{array}{c}73,40 \mathrm{~b} \\
(0,51)\end{array}$ & $\begin{array}{c}18,41 \mathrm{~b} \\
(3,31)\end{array}$ \\
\hline E. grandis & $\begin{array}{c}71,04 \mathrm{~b} \\
(3,57) \\
\end{array}$ & $\begin{array}{c}11,13 \mathrm{c} \\
(2,08)\end{array}$ & $\begin{array}{c}22,36 \mathrm{a} \\
(0,34)\end{array}$ & $\begin{array}{c}24,83 \mathrm{~b} \\
(1,52) \\
\end{array}$ & $\begin{array}{c}63,07 \mathrm{a} \\
(3,42) \\
\end{array}$ & $\begin{array}{c}13,53 \mathrm{a} \\
(3,31) \\
\end{array}$ \\
\hline P. elliotti & $\begin{array}{c}81,89 \mathrm{c} \\
(3,24)\end{array}$ & $\begin{array}{l}3,71 \mathrm{a} \\
(0,43) \\
\end{array}$ & $\begin{array}{c}24,47 \mathrm{c} \\
(1,70)\end{array}$ & $\begin{array}{c}24,80 \mathrm{~b} \\
(1,77)\end{array}$ & $\begin{array}{c}81,29 \mathrm{c} \\
(1,00)\end{array}$ & - \\
\hline $\mathrm{F}$ & $109,78 *$ & $109,60 *$ & $61,13 *$ & $43,71 *$ & 217,78 & 179,22 \\
\hline CV $(\%)$ & 10,78 & 3,40 & 11,73 & 11,70 & 10,78 & 77,52 \\
\hline \multicolumn{7}{|c|}{ FACE RADIAL } \\
\hline AMOSTRAS & $L^{*}$ & $A^{*}$ & $\mathrm{~B}^{*}$ & $\mathrm{C}^{*}$ & $\mathrm{H}^{\circ}$ & \\
\hline
\end{tabular}




\begin{tabular}{c|c|c|c|c|c|c}
\hline \multirow{2}{*}{ C. maculata } & $66,29 \mathrm{a}$ & $5,44 \mathrm{a}$ & $20,23 \mathrm{a}$ & $20,98 \mathrm{a}$ & $74,85 \mathrm{~b}$ & $17,08 \mathrm{~b}$ \\
& $(1,22)$ & $(0,50)$ & $(0,240)$ & $(0,92)$ & $(1,08)$ & $(1,34)$ \\
\hline \multirow{2}{*}{ E. grandis } & $72,48 \mathrm{~b}$ & $11,81 \mathrm{~b}$ & $23,15 \mathrm{~b}$ & $26,02 \mathrm{~b}$ & $63,18 \mathrm{a}$ & $12,69 \mathrm{a}$ \\
& $(3,74)$ & $(2,03)$ & $(1,44)$ & $(1,52)$ & $(2,89)$ & $(3,70)$ \\
\hline \multirow{2}{*}{ P. elliotti } & $81,20 \mathrm{c}$ & $5,09 \mathrm{a}$ & $27,12 \mathrm{a}$ & $27,60 \mathrm{~b}$ & $79,38 \mathrm{c}$ & - \\
& $(2,30)$ & $(0,76)$ & $(3,20)$ & $(1,77)$ & $(0,92)$ & 182,53 \\
\hline $\mathrm{F}$ & $97,12 *$ & $103,27 *$ & $32,51 *$ & $25,89 *$ & 243,42 & 77,29 \\
\hline
\end{tabular}

Em que: valores entre parênteses junto às médias representam o desvio padrão do tratamento e letras diferentes, nas colunas, indicam diferenças significativas conforme o teste LSD Fisher, em 5\% de probabilidade de erro; - variação dos testes de colorimetria; $L^{*}$-claridade; $a^{*}$-coordenada vermelho-verde;-coordenada azul-amarelo; $C^{*}$ - saturação da cor; $h^{\circ}$ ângulo de tinta; CV (\%) - coeficiente de variação dos tratamentos; DP - desvio padrão dos tratamentos; * - apresenta diferença significativa pelo teste $\mathrm{F}(\mathrm{p}<0,05)$; ns - não apresenta diferença significativa pelo teste $\mathrm{F}(\mathrm{p} \geq 0,05)$.

De acordo com a coordenada L* (luminosidade esta varia de 0 (preto) à 100 (branco), Já coordenada cromática a* (vermelho-verde) caracteriza madeiras com tonalidades vermelhas (+) ou com características de tonalidades verdes (-), onde as alterações nos valores dessa variável, quando comparadas com o grupo controle, indicam a diferenças da constituição química [27].

Para a coordenada a* (espectro do vermelho), observa-se pelas Tabelas 5 que a madeira de P. elliottii apresentou menores valores. Isso significa que esta espécie tende a ser mais esbranquiçada que as demais analisadas. Com significado inverso, observa-se que o maior valor da coordenada a* na espécie de E grandis, caracterizando-a como uma espécie com uma tonalidade mais acentuada na tonalidade vermelha se comparado as demais analisadas. Analisando a coordenada azul-amarelo $\left(b^{*}\right)$, quando for sinal positivo indica madeiras com tonalidade amarela, enquanto o sinal negativo tende a tonalidade azul, a saturação $-\mathrm{C}^{*}$ quanto menor o valor mais opaco e o ângulo de tinta $-\mathrm{h}^{\circ}$ mais próximo a $0^{\circ}$ tendência á vermelho e mais próximo de $90^{\circ}$ á amarelo.

Nota-se que para a coordenada cromática $\mathrm{b}^{*}$ apresenta maior valores para a espécie $P$. elliottii e o menor valor para C.maculata, o que retoma a espécie de $P$. elliottii uma tonalidade mais amarelada enquanto a C.maculata uma tonalidade marrom-escuro. Moura [28] e Modes [18], ao analisarem as propriedades colorimétricas damadeira de Pinus e Eucalyptus, constatou uma coloração no grupo controle de amarela-esbranquiçada para a madeira de Pinus e avermelhada com tonalidade clara para madeira de Eucalyptus.

Em relação a saturação $C^{*}$ (coordenada que corresponde a distribuição colorimétrica) os maiores valores também são demostrados para a madeira de $P$. elliottii o que demostra que esta possui maior homogenidade colorimétrica, ou seja, melhor distribuição da cor, seguida por E. grandis e C. maculata. O ângulo de tinta $\mathrm{h}^{\circ}$ é diretamente relacionado as coordenadas cromáticas $\mathrm{a}^{*} \mathrm{e} \mathrm{b}^{*}$, visto que estas variaram entre as espécies então o ângulo de tinta $\mathrm{h}^{\circ}$ tanto para o plano anatômico tangencial como radial demostraram diferenças significativas.

Para o (variação total da cor das madeiras), foi utilizado como controle a espécie P.elliottii pois esta possui tendência visual em ser a madeira mais clara dentre as estudadas, observa-se que a maior variação em relação ao controle, se obteve com a espécie C.maculata, tal variação é dependente das coordenadas L*, a* e b*, visto que há uma maior variação entre as mesmas.

A análise dos espectros de FT-IR para a madeira, Figura 1, é melhor representada na região entre 1800 e $600 \mathrm{~cm}^{-1}$, mais conhecida como impressão digital, em que cada vibração em um determinado número de onda, representa um tipo de ligação que se relaciona com os componentes químicos majoritários da madeira. Popescu et al. [29], indica que em regiões onde as vibrações predominantes são dos grupos $\mathrm{OH}$ e $\mathrm{CH}$, entre elas a região entre $2000 \mathrm{e} 800 \mathrm{~cm}^{-1}$, onde há vibração de determinado grupo, pode então ser atribuída possivelmente a presence de maiores quantidades de grupos hidroxilas. 


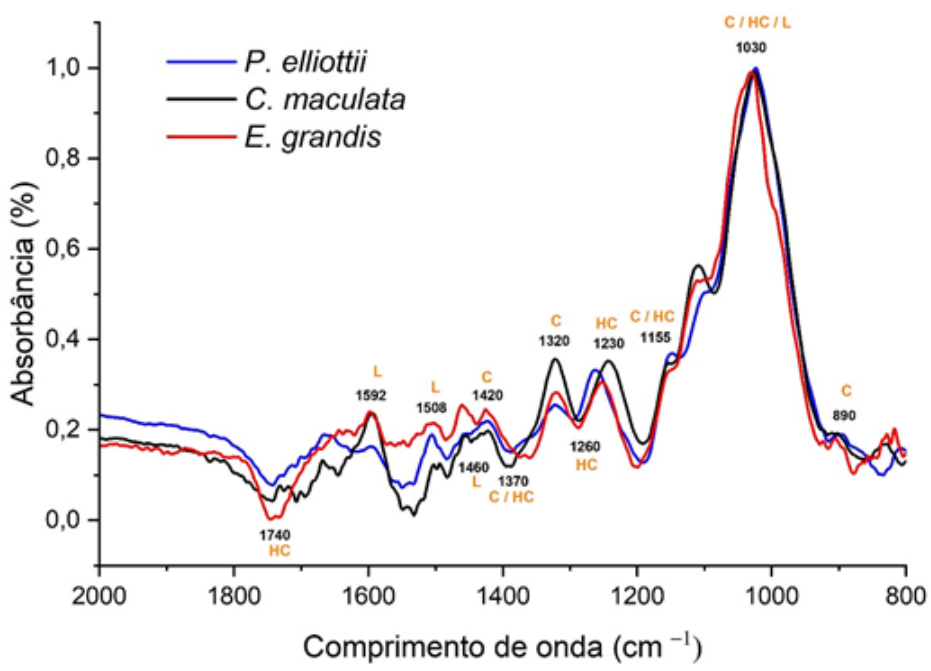

Figura 1: Espectros obtidos através de análise FT-IR.

Em que: $\mathrm{C}$ - celulose, $\mathrm{HC}$ - hemicelulose e L - lignina.

As maiores variações entre as espécies foram evidenciadas em destaque nas bandas de $1508 \mathrm{~cm}^{-1}, 1592 \mathrm{~cm}^{-1}, 1320$ $\mathrm{cm}^{-1}$ e $1740 \mathrm{~cm}^{-1}$ sendo estes picos caracterizados por $\mathrm{C}=\mathrm{C}$ alongamento vibração em anel aromático, vibrações aromáticas com alongamento $\mathrm{C}=\mathrm{O}$, vibrações no grupo $\mathrm{CH}_{2}$ e variação nas bandas de $\mathrm{C}=\mathrm{O}$ do grupo carboxílico, sucessivamente [30, $31,32,33]$.

As bandas $1508 \mathrm{~cm}^{-1} \mathrm{e} 1592 \mathrm{~cm}^{-1}$, está relacionado ao componente químico majoritário da lignina, onde o maior pico é correspondente a espécie E.grandis.Segundo Evans [34] intensidades das bandas de vibração esqueléticas aromáticas encontradas em tais regiões. analiando madeiras com menor resistência mecânica a banda a $1592 \mathrm{~cm}^{-1}$ é significativamente menos intensa do que a de $1508 \mathrm{~cm}^{-1}$, nas madeiras com maior resistência mecânica, ocorre o contrário, indo de acordo com o presente estudo, que mostra um pico mais intenso para a madeira de C.maculata tendênciando que esta espécie possui ligações mais fortes nesta banda. -

Quando analisando a banda de $1320 \mathrm{~cm}^{-1}$ é predominante os picos para a espécie de C.maculata e menor absorbância para a espécie de P.elliottii é caracterizada por maior frequência de ocorrência de ligações nos grupos das celuloses. A banda de $1740 \mathrm{~cm}^{-1}$ caracteriza maior frequência em ligações do grupo das hemiceluloses, conforme Chen [35] está banda, do ponto de vista mecânico, caracteriza uma relação entre a dureza, quanto maior a intensidade do pico maior será a dureza da mesma, justificando o apresentado na Tabela 2 onde a espécie com maior dureza foi a de C.maculata.

A banda de $1030 \mathrm{~cm}^{-1}$ é característica de ligações $\mathrm{C}-\mathrm{O}$ alongamento e deformação de $\mathrm{C}-\mathrm{H}$ na unidade de guaiacil, representando por mudanças nos grupos químicos da celulose, hemiceluloses e ligninas. Darwish et al. [36], esta banda possuir a maior absorbância é típico de todo tipo de madeira, considerando a maior frequência de ocorrência deste tipo de ligação de modo geral nas três espécies analisadas. Estas diferenças espectrais resultantes do FTIR, pode ser usadas para auxiliar a descrever características mecânicas de qualquer tipo de madeira [37].

As curvas termogravimétricas (TGA) trazem a representação da perda de massa, em porcentual, em relação a temperatura, enquanto as curvas DTG fazem correspondências à primeira derivada das curvas do TGA. Na Figura 2 são demostradas as curvas termogravimétricas (TGA/DTG) das 3 espécies analisadas, na faixa de temperatura de 0 a $600{ }^{\circ} \mathrm{C}$.
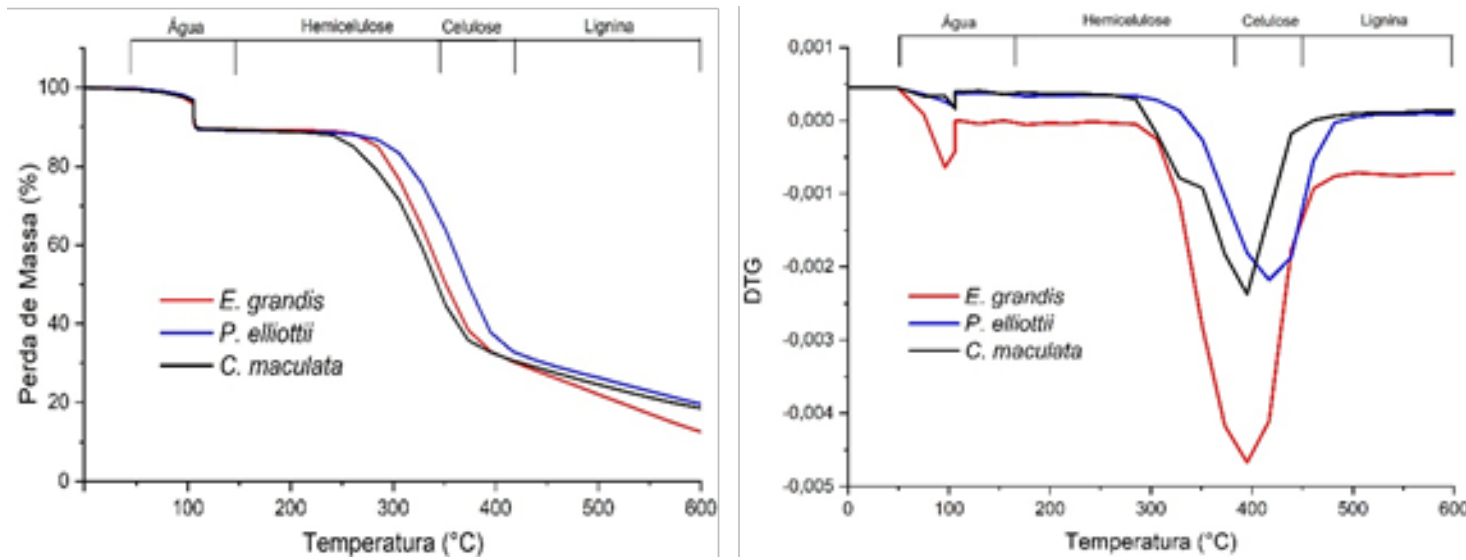

Figura 2: Curvas de análise termogravimétrica (TGA) e (DTG). 
O percentual de perda de massa em relação a temperatura que esse ocorre é dividido em 4 faixas características, sendo estas: $40-150^{\circ} \mathrm{C}$ para remoção da água inter e intracelular presente na madeira, $150-380^{\circ} \mathrm{C}$ degradação das hemiceluloses, $380-420^{\circ} \mathrm{C}$ degradação das celuloses e $420^{\circ} \mathrm{C}$ em diante, degradação das ligninas presentes na madeira.

Em relação a faixa de degradação das hemiceluloses esse apresentou picos máximos de degradação nas faixas de $400^{\circ} \mathrm{C}$, a espécie de $E$. grandis demostrando o maior percentual de DTG. Pereira [38] ao analisar a estabilidade térmica de 6 clones do gênero Eucalyptus, notou uma degradação máxima na faixa dos $390^{\circ} \mathrm{C}$, isso ocorre em função das hemiceluloses serem os primeiros componentes majoritários da madeira a se degradarem [39].

A faixa de degradação das celuloses (faixa $400-450^{\circ} \mathrm{C}$ ) demostra maiores valores de pico para E.grandis, P. elliottii e C. maculata sucessivamente, o que indica que a madeira de E. grandis possui maior degradação da celulose que as demais espécies analisadas.

A lignina possui resistência térmica superior aos demais componentes da madeira. Sua degradação térmica está situada entre $225^{\circ} \mathrm{C}$ e $450^{\circ} \mathrm{C}$. Em torno de $200{ }^{\circ} \mathrm{C}$, segundo Schniewind [40], ocorrem reações de desidratação. Entre 150 ${ }^{\circ} \mathrm{C}$ e $300{ }^{\circ} \mathrm{C}$ ocorre o rompimento das ligações de carbono e, em torno de $300{ }^{\circ} \mathrm{C}$, as ligações laterais alifáticas começam a romper fora do anel aromático. O pico com maior intensidade de degradação se deu na espécie de E. grandis, as espécies de C. maculata de P. elliottii mostraram resultados semelhantes em relação a degradação deste componente.

Percebe-se que a espécie de P.elliottie C.maculata, se mostraram mais estável termicamente, quando comparada ao E.grandis, possuindo um menor percentual de perda de massa e um retardo de temperatura em relação ao pico máximo de degradação. Tal fator pode estar associado a madeira de P.elliotti ser uma conifera e portanto apresentar menores percentuais de holocelulose (hemicelulose + celulose), quando comparado a folhosas. Analisando a C.maculata por apresentar uma elevada densidade, possui maior percentual de massa por unidade de volume, portanto uma intensidade menor em relação ao pico máximo de degradação residual, quando comparado a espécie de E.grandis.

De acordo com Santos [41], a espessura de parede das fibras influencia indiretamente na conversão da madeira em carvão vegetal e diretamente para a massa especifica, pois, uma madeira com fibras de parede celular espessa apresentará maior volume de biomassa para sustentar a degradação térmica da madeira. Figueroa [42], relata que o amplo conhecimento da perda de massa e das reações endotérmicas e exotérmicas em função da temperature, possibilita considerar a variação da massa específica em função da temperatura e, também, as fontes de massa de geração de calor nas mesmas temperaturas que ocorrem as reações exotérmicas.

\section{CONCLUSÕES}

A partir dos valores médios das propriedades físicas e mecânicas das três espécies florestais analisadas, foi verificado variações significativas, onde a espécie com maior densidade (C. maculata) demostrou melhor comportamento físico-mecânico.

A espectroscopia de infravermelho com transformada de Fourier (FT-IR) demonstrou ser uma ferramenta eficiente, quando analisado a variabilidade da composição química entre as três espécies florestais estudadas, com potencial para correlação entre as propriedades químicas e mecânicas.

Em relação ao TGA/DTG as espécies de P.elliotti e C.maculatta, se mostraram mais estável termicamente, quando comparada ao E. grandis, possuindo um menor percentual de perda de massa e um retardo de temperatura em relação ao pico máximo de degradação.

Sendo assim, destaca-se uma heterogeneidade entre as espécies avaliadas nesse estudo, tanto do ponto de vista físico, mecânico, térmico e químico, fazendo-se necessário a caracterização de tais propriedades para o emprego correto das mesmas.

\section{BIBLIOGRAFIA}

[1] NEIVA, D. et al. "Composição Química e Potencial de Polpação Kraft de 12 Espécies de Eucalipto". Culturas e Produtos Industriais, v. 66, pp. 889-95, 2015.

[2] BALLESTEROS, J.E.M., et al. "Evaluation of the Effect of Drying and Rewetting Cycles in Eucalyptus Pulps". International Journal of Engineering and Technology, v. 7, n. 5, pp. 397-400, 2015.

[3] MÜLLER, B.V., ROCHA, M.P., CUNHA, A.B., el al. "Avaliação das principais propriedades físicas e mecânicas da madeira de Eucalyptus benthamii Maiden et Cambage”. Floresta e Ambiente, v. 21, n. 4, pp. 535-542, 2014.

[4] LAHR, F.A.R., et al. "Cisalhamento e Módulo Longitudinal de Elasticidade em Madeira: relações baseadas em testes de flexão estática”. Acta Scientiarium Technology, v. 39, n. 4, pp. 433-437, 2017. 
[5] PYÖRÄLÄ, J. et al. Variability of wood properties using airborne and terrestrial laser scanning. Remote Sensing of Environment, v.235, pp. 14, 2019.

[6] MORI, C.L.S.O., LIMA, J.T., MORI, F.A., el al. "Caracterização da cor da madeira de clones de híbridos de Eucalyptus spp". Cerne, v. 11, n. 2, pp. 137-146, 2005.

[7] CARNEIRO, A.D.C.O., SANTOS, R.C., CASTRO, R.V.O., et al. "Estudo da decomposição térmica da madeira de oito espécies da Região do Seridó, Rio Grande do Norte”. Revista Árvore, v. 37, n. 6, pp. 1153-1163, 2013.

[8] GALLIO, E., ZANATTA, P., RIBES, D.D., et al. "Fourier transform infrared spectroscopy in treated woods deteriorated by a white rot fungus". Maderas Ciencia y tecnología, v. 20, n. 3, pp. 479-488, 2018.

[9] OJEDA, J.J., DITTRICH, M. Fourier transform infrared spectroscopy for molecular analysis of microbial cells. Methods in molecular biology, v.881, pp. 187-211, 2012.

[10] ASTM - AMERICAN SOCIETY FOR TESTING AND MATERIALS. D2395-17. "Standard Test Methods for Density and Specific Gravity (Relative Density) of Wood and Wood-Based Materials”, Philadelphia, 2017.

[11] ASTM - AMERICAN SOCIETY FOR TESTING AND MATERIALS. D143-94: "Standard test methods for small clear specimens of timber", Philadelphia, 2014.

[12] MARTINS, M., SILVA, J.R.M.D., LIMA, J.T., et al. "Simulação em uso dos pisos de madeira de Eucalyptus sp e Corymbia maculata”. Cerne, v. 19, n. 1, pp. 151-156, 2013.

[13] GONÇALEZ, J.C. et al. "Características Tecnológicas das madeiras de Eucalyptus grandis W.Hill ex Maiden e Eucalyptus cloeziana F. Muell visando ao seu aproveitamento na indústria moveleira”. Ciência Florestal, Santa Maria, v. 16, n. 3, pp. 329-341, 2006.

[14] SILVA, J.C. et al. "Variação da retratibilidade da madeira de Eucalyptus grandis Hill ex Maiden, em função da idade e da posição radial no tronco". Revista Árvore, Viçosa, v. 30, n. 5, pp. 803-810, 2006.

[15] BATISTA, D.C., KLITZKE, R.J., SANTOS, C.V.T. "Densidade básica e retratibilidade da madeira de clones de três espécies Eucalyptus”. Ciência Florestal, v.20, n. 4, pp. 665-674, 2010.

[16] VIDAURRE, G., LOMBARDI, L.R., OLIVEIRA, J.D.S., et al. "Lenho juvenil e adulto e as propriedades da madeira”. Floresta e Ambiente, v. 18, n. 4, pp. 469-480, 2011.

[17] XAVIER, R.B.L. Avaliação da dureza janka, densidade e estabilidade quatro espécies de eucalyptus implantadas no estado do rio de janeiro. UFRJ. Monografia. Rio de Janeiro, Brasil, pp. 22, 2008.

[18] MODES, K.S., SANTINI, E.J., VIVIAN, M.A., et al. "Efeito da termorretificação nas propriedades mecânicas das madeiras de Pinus taeda e Eucalyptus grandis”. Ciência Florestal, v. 27, n.117, pp. 291-302, 2017.

[19] GONÇALVES F. G, OLIVEIRA J.T.S, LUCIA R.M.D, SARTÓRIO R.C. "Estudo de algumas propriedades mecânicas da madeira de um híbrido clonal de Eucalyptus urophylla x Eucalyptus grandis". Árvore, v. 33, n. 3, pp. 501-509, 2009.

[20] GONÇALEZ, J. C., et al. "Influência do sítio nas propriedades da madeira de Pinus caribaea var. hondurensis". Cerne, v. 15, n. 2 , pp. 251-255, 2009.

[21] VEIGA, T.R.L.A., LIMA, J.T., MONTEIRO, T.C., el al."Propriedades mecânicas de amostras individualizadas da madeira e do carvão de Eucalyptus urophylla e de Corymbia citriodora". Scientias Forestalis, v. 46, n. 117, pp. 107-114, 2018.

[22] LEITE, M. K. Caracterização tecnológica da madeira de Corymbia maculata, Eucalyptus cloeziana e E. resinifera para a aplicação no design de Produtos de Maior Valor Agregado (PMVA). Tese de D. Sc., USP, São Paulo, SP, Brasil, pp. 135, 2013.

[23] HASELEIN, C.R., BERGER, R., GOULART, M., et al. "Propriedades de flexão estática da madeira úmida e a 12\% de umidade de um clone de Eucalyptus saligna Smith sob o efeito do espaçamento e da adubação". Ciência Florestal, Santa Maria, v. 12, n. 2, pp.147- 152, 2002.

[24] LOBÃO, M.S. et al. "Caracterização das propriedades físico-mecânicas da madeira de eucalipto com diferentes densidades”. Revista Árvore, v. 28, n. 6, pp. 889-894, 2004.

[25] LIMA, I.L., GARCIA, J.N. "Variação da densidade aparente e resistência a compressão paralela às fibras em função da intensidade de desbaste, adubação e posição radial em Eucalyptus grandis Hill Ex-Maiden”. Árvore, v. 34, n. 3, pp. 551-559, 2010.

[26] SERPA, P.N. et al. "Evaluation of some properties of Eucalyptus grandis, Eucalyptus saligna and Pinus elliottii". Revista Árvore, v. 27, n. 5, pp. 723-733, 2003. 
[27] ZANUNCIO, A. J. V., DE SÁ FARIAS, E., DA SILVEIRA, T. A. “Termorretificação e colorimetria da madeira de Eucalyptus grandis". Floresta e Ambiente, v. 21, n. 1, pp. :85-90, 2014.

[28] MOURA, L. F., BRITO, J. O. "Effect of thermal rectification on colorimetric properties of Eucalyptus grandis and Pinus caribaea var. hondurensis woods". Scientia Forestalis, v. 39, n. 89, pp. 69-76, 2011.

[29] POPESCU, C., POPESCU, M., VASILE, C. "Characterization of fungal degraded lime wood by FT-IR and 2D IR correlation spectroscopy”. Microchemical Journal,v.95, pp. 377-387, 2010.

[30] POZO, C., DÍAZ-VISURRAGA, J., CONTRERAS, D., et al."Characterization of temporal biodegradation of radiata pine by Gloeophyllum trabeum through principal component analysis-based two-dimensional correlation FTIR spectroscopy". Journal of the Chilean Chemical Society, v. 61, n. 2, pp. 2878-2883, 2006.

[31] COSTA, M., COSTA, A. F., PASTORE, T. C. M., et al. "Caracterização do ataque de fungos apodrecedores de madeiras através da colorimetria e da espectroscopia de infravermelho". Ciência Florestal, v.21, n.3, pp. 567-577, 2011.

[32] SUN, B., HUANG, A., WANG, Y., LIU, J. "Natural bamboo (Neosinocalamus affinis Keng) fiber identification using FT-IR and 2D-IR correlation spectroscopy". Journal of Natural Fibers, v. 12, n. 1, pp. 1-11, 2015.

[33] ZHANG, X., WANG, F., KEER, L. M. "Influence of surface modification on the microstructure and thermo-mechanical properties of bamboo fibers”. Materials, v.8, pp. 6597-6608, 2015.

[34] EVANS, P. A. "Differentiating "hard" from "soft" woods using Fourier transform infrared and Fourier transform spectroscopy”. Spectrochimica Acta Part A: Molecular Spectroscopy, v. 47, pp. 1441-1447, 1991.

[35] CHEN, HUILUN et al. "Qualitative and quantitative analysis of wood samples by Fourier transform infrared spectroscopy and multivariate analysis". Carbohydrate polymers, v. 82, n. 3, pp. 772-778, 2010.

[36] DARWISH, S. S., EL HADIDI, N. M. N., MANSOUR, M. "The effect of fungal decay on Ficus sycomorus wood". International Journal of Conservation Science, v. 4, n. 3, pp. 271-282, 2013.

[37] TOIVANEN, T.J., ALEN, R. A. "FTIR/PLS method for determining variations in the chemical composition of birch (Betula pendula/B-publescens) stem wood”.Appita Journal, v. 60, pp. 155-160, 2007.

[38] PEREIRA, C. B. L., CARNEIRO, A. C. O., CARVAlHO, A. M. M. L., et al. "Estudo da degradação térmica da madeira de Eucalyptus através de termogravimetria e calorimetria”. Revista Árvore, v. 37, n. 3, pp. 567-576, 2013.

[39] FTA - FINNISH THERMOWOOD ASSOCIATION. “ThermoWood ${ }^{\circledR}$ handbook”. Helsinki, pp. 66, 2003.

[40] SCHNIEWIND, A. P. “Concise Encyclopedia of Wood and Wood-Based Materials". Nova York: Pergamon Press, pp. 271-273, 1985.

[41] SANTOS, R.C., CARNEIRO, A.C.O., CASTRO, A.F.M., et al. Correlações entre os parâmetros de qualidade da madeira e do carvão vegetal de clones de eucalipto. Scientia Forestalis, Piracicaba, v. 39, n. 90, pp. 221-230, 2011.

[42] FIGUEROA, M. J. M., DE MORAES, P. D. “Comportamento da madeira a temperaturas elevadas". Ambiente construido, v. 9, n. 4, pp. 157-174, 2009.

\section{ORCID}

Henrique Römer Schulz

https://orcid.org/0000-0002-8162-7723

Andrey Pereira Acosta

https://orcid.org/0000-0002-5074-3772

Ezequiel Gallio

https://orcid.org/0000-0002-0603-1065

Kelvin Techera Barbosa

https://orcid.org/0000-0001-9894-703X

Darci Alberto Gatto

https://orcid.org/0000-0002-6805-3243 
